\title{
Structural and Chemical Analyser for SEM studies of nanostructures
}

\author{
Dr.A.D.Brooker*, Dr.J.W.Baldwin**, Dr.P.E.Pehrssohn**, Dr.James.E.Butler** \\ * Renishaw PLC, Old Town, Wotton-under-edge, Glos., GL12 7DW, UK \\ ** Naval Research Laboratory, Chemistry Division, 4555 Overlook Ave. DC 20375
}

This paper describes a new analytical method - the structural and chemical analyser (SCA) - for scanning electron microscopes (SEMs), and its application to the study of nanostructures. In order to collect Raman, photoluminescence, and cathodoluminescence spectra whilst observing a secondary electron image, an SEM interface that can be connected to a spectrometer has been constructed. Using the SCA it is now possible to determine the chemical, structural, mechanical, and electrical properties of various materials at the same spatial resolution as conventional X-ray analysis (or better), without having to move the sample between multiple instruments. The SCA interface can be fitted to many different SEM makes and models, and the nature of Raman spectroscopy means that its performance is not affected by the operating environment making it compatible with ESEM, LVSEM, HV-SEM, FEG-SEM and even UHV systems.

Integrating an optical spectroscopy method with SEM required the development of novel technologies to overcome design constraints, and to meet the desired performance of the system. In particular to achieve effective analysis of micrometre-sized objects, then the collection optics need to be repeatably positioned with sub-micron accuracy, and customised optics are needed to meet vacuum requirements, and to preserve analytical spot size and light collection efficiency and wavelength range.

Conventional in-SEM analytical methods have only limited materials characterisation capability. Energy-dispersive x-ray analysis (EDS) for example is fast, easy to use, and easy to interpret and quantify, but it provides only elemental information - chemical identification of the detected species can only be assumed or calculated quantitatively using stoichiometry estimations. Furthermore, $\mathrm{x}$ ray detectors have poor sensitivity to light elements. Raman spectroscopy, however, yields both chemical and structural information about samples, and is highly sensitive to light element chemistry.

Similarly conventional Raman spectroscopy can often be hampered by the poor depth of field and lack of contrast of optical microscopy methods, whereas SEM has excellent depth of field, and numerous contrast mechanisms that can distinguish between optically similar materials. Additionally SEM has much higher spatial resolution enabling the visualisation of structures beyond the resolution limit of optical microscopy.

The design capabilities of the new analytical technique are briefly discussed, and these are compared and contrasted with those of the conventional in-SEM analytical methods, and with conventional optical microscope-based Raman spectroscopy.

The Structural and Chemical analyser used Raman spectroscopy to examine bulk and discrete carbon nanotubes in an SEM. Figures 1 and 2 compare the in-SEM and OM analysis of the bulk nanotubes using 532 and $633 \mathrm{~nm}$ laser excitation and demonstrate clearly the resonance enhancement effect of the tangential phonon and radial breathing modes (seen between 1500-1600 $\mathrm{cm}^{-1}$ and 130$300 \mathrm{~cm}^{-1}$ Raman shift respectively) described by Pimenta and Marrucci et al [1]. 
SEM observation was used to confirm the presence, and individual nature of single-wall carbon nanotubes that had been deposited between electrodes on a silicon substrate (see Figure 3), and Raman spectroscopy was used to analyse them. The Raman spectra from bulk and individual nanotubes reported in Figure 4 correlate closely with data collected by Dresselhaus and Eklund [2].

${ }^{1}$ M.A.Pimenta \& A.Marrucci et al, Phys Rev B, 58, 24, (1998), R16 016-019

${ }^{2}$ M.S.Dresselhaus \& P.C.Eklund, Advances in Physics, 49, 6, (2000), 705-814

${ }^{3}$ Renishaw plc wishes to thank the Chemistry Division of the Naval Research Laboratory for the use of their equipment, and provision of the samples

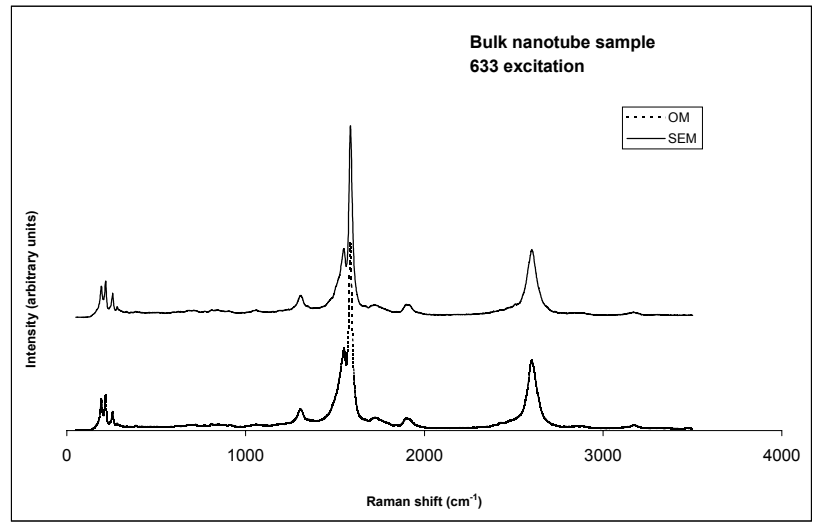

Figure 1 - Raman spectra showing 633nm excited Raman spectra from bulk nanotubes inSEM and using OM

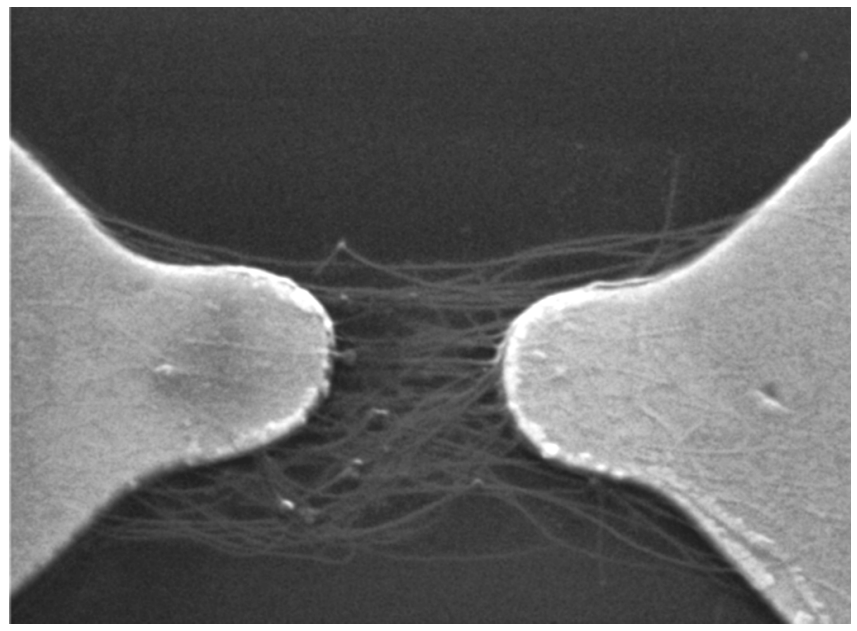

Figure 3 - SEM image showing Carbon Nanotubes aligned between gold electrodes. The gap between the electrodes is $1 \mu \mathrm{m}$

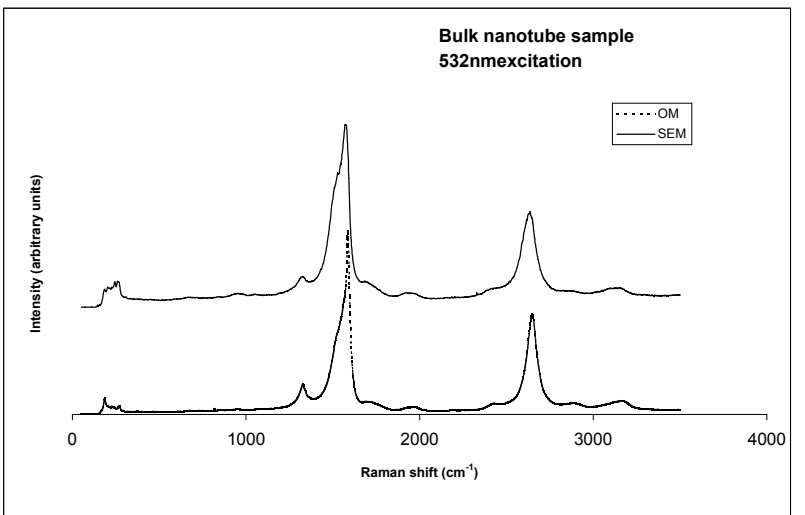

Figure 2 - Raman spectra showing 633nm excited Raman spectra from bulk nanotubes inSEM and using OM

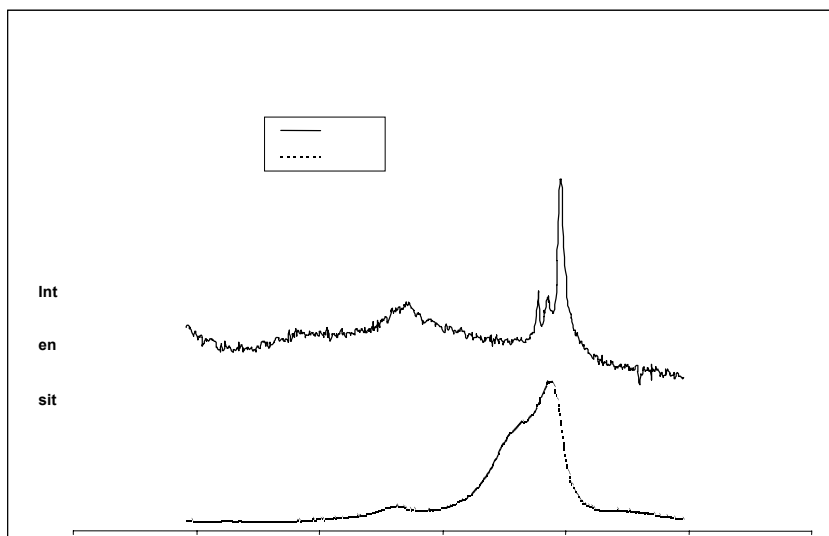

Figure 4 - Raman spectra showing the tangential phonon mode bands for discrete and bulk singlewalled nanotubes 